\title{
Stability analysis for discrete-time fractional-order LTI state-space systems. Part I: New necessary and sufficient conditions for the asymptotic stability
}

\author{
R. STANISŁAWSKI and K.J. LATAWIEC** \\ Institute of Control and Computer Engineering, Opole University of Technology, 31 Sosnkowskiego St., 45-272 Opole, Poland
}

\begin{abstract}
This paper presents a series of new results on the asymptotic stability of discrete-time fractional difference (FD) state space systems and their finite-memory approximations called finite FD (FFD) and normalized FFD (NFFD) systems. In Part I, new, general, necessary and sufficient stability conditions are introduced in a unified form for FD/FFD/NFFD-based systems. In Part II, an original, simple, analytical stability criterion is offered for FD-based systems, and the result is used to develop simple, efficient, numerical procedures for testing the asymptotic stability for FFD-based and, in particular, NFFD-based systems. Consequently, the so-called $f$-poles and $f$-zeros are introduced for FD-based system and their closed-loop stability implications are discussed.
\end{abstract}

Key words: Grünwald-Letnikov fractional difference, discrete-time fractional-order systems, stability conditions, Cauchy's argument principle.

\section{Introduction}

Non-integer or fractional-order dynamic models have recently attracted a considerable research interest. Their specific properties can make them more adequate in modeling of selected industrial systems [1-4]. However, an infinite-memory filter incorporated in a fractional difference (FD) may lead to a computational explosion. Therefore, a number of discrete-time fractional difference (FD) LTI systems have been modeled both via transfer function or difference equation models [5-9] and state space ones [8,10-12].

Apart from the computational and accuracy aspects in approximating FD-based systems [13], our main reservation against the above recalled approximation approaches based on infinite impulse response (IIR) / finite impulse response (FIR) / orthonormal basis function (OBF) filters is that they are quite arbitrary, in that they do not use any a priori knowledge about the mathematical (not to say physical) structure of FD [13]. Therefore we advocate an alternative approach relying on the approximation of the FD filter with its truncated, finite-memory version $[10,11,14-16]$. In analogy to FIR, the term finite FD, or FFD, has been coined [13,17-19]. FFD may however suffer from (remarkable) steady-state errors with respect to FD [13]. To cope with this, we have introduced what is here referred to as normalized FFD or NFFD [13, 18, 19]. In particular, in Ref. [13,18], computational and steady-state accuracy aspects for FFD and NFFD have been considered and some results are recalled here.

Unlike for continuous-time LTI systems [20-25], stability analysis for fractional order discrete-time LTI state space systems has not been given due research attention. First stability results for FFD-based systems have included either (analytical) sufficient conditions only $[10,15]$ or numerically in- efficient necessary and sufficient ones [10,15,26,27]. Later complete analytical results for FD/FFD-based systems have been obtained for a specific case of positive systems only $[11,14,28-31]$. Up to date, no general, either analytical or numerically effective stability criteria have been given for arbitrary FD/FFD/NFFD-based systems. In particular, it is not until this paper that the continuous-time stability criterion of Matignon [23] is extended to discrete-time fractional-order systems.

This paper presents a number of new results concerning the stability analysis for FD, FFD and NFFD-based LTI state space systems. In particular, necessary and sufficient stability conditions for the systems are offered in a general, unified, FD/FFD/NFFD-based form in Part I. This gives rise to the culmination in Part II with an original, simple stability criterion for FD-based systems and simple numerical procedures for testing the stability of FFD and NFFD-based systems. Consequently, new definitions of poles and zeros of FD-based systems, called $f$-poles and $f$-zeros, are offered, which are used in the stability analysis of closed-loop systems.

Part I of the paper is structured as follows. Having introduced the FD modeling problem in Sec. 1, the GrünwaldLetnikov fractional discrete-time difference is recalled, together with its FFD and NFFD approximations, in Sec. 2. An application of FFD/NFFD in modeling of state-space systems is presented in Sec. 3, with steady-state accuracy results recalled. The most important Sec. 4 gives new, general, necessary and sufficient conditions for the stability of NFFD and FFD-based systems as well as FD-based ones, all finally presented in a unified form of Theorem 7. Conclusions of Sec. 5 summarize the achievements of the first part of the paper.

*e-mail: k.latawiec@po.opole.pl 


\section{Fractional difference}

A simple generalization of the familiar Grünwald-Letnikov difference [32] is the fractional difference (FD) in discrete time $t=0,1, \ldots$, described by equation $[11,12,15,33]$

$$
\begin{aligned}
& \Delta^{\alpha} x(t)=\sum_{j=0}^{t} P_{j}(\alpha) x(t) q^{-j} \\
& =x(t)+\sum_{j=1}^{t} P_{j}(\alpha) x(t) q^{-j},
\end{aligned}
$$

where $\alpha \in(0,2)$ is the fractional order, $q^{-1}$ is the backward shift operator and

$$
P_{j}(\alpha)=(-1)^{j} C_{j}(\alpha),
$$

with

$$
C_{j}(\alpha)=\left(\begin{array}{c}
\alpha \\
j
\end{array}\right)= \begin{cases}1 & j=0 \\
\frac{\alpha(\alpha-1) \ldots(\alpha-j+1)}{j !} & j>0\end{cases}
$$

Note that each element in Eq. (1) from time $t$ back to 0 is nonzero so that each incoming sample of the signal $x(t)$ increases the complication of the model equation. In the limit, with $t \rightarrow+\infty$, we end up with computational explosion.

Remark 1. Possible accounting for the sampling period $T$ when transferring from a continuous-time derivative to the discrete-time difference results in dividing the right-hand side of Eq. (1) by $T^{\alpha}$ [15]. Operating without $T^{\alpha}$ as in the sequel corresponds to putting $T=1$ or to the substitution of $P_{j}(\alpha)$ for $\frac{P_{j}(\alpha)}{T^{\alpha}}, j=0, \ldots, t$.

2.1. Finite fractional difference. In [13, 17-19], truncated or finite fractional difference (FFD) has (in analogy to FIR) been considered for practical, feasibility reasons, with the convergence to zero of the series $C_{j}(\alpha)$ enabling to assume $C_{j}(\alpha) \approx 0$ for some $j>\bar{J}$, where $\bar{J}$ is the number of backward signal samples used to calculate the fractional difference. A formal definition of FFD has been presented in $[13,18]$ as follows

Definition 1. Let the fractional difference (FD) be defined as in Eqs. (1) to (3). Then the finite fractional difference (FFD) is defined as

$$
\Delta^{\alpha} x(t, J)=x(t)+\sum_{j=1}^{J} P_{j}(\alpha) x(t) q^{-j},
$$

where $J=\min (t, \bar{J})$ and $\bar{J}$ is the upper bound for $j$.

The FFD has been analyzed in some papers under the heading of a practical implementation of FD [11,14], or a finite difference $[10,15]$, or a short-memory difference [16].

An important problem encountered in FFD-based modeling is an incorrect steady-state gain of the model, with its discrepancy with respect to the corresponding FD one being dependent on $\bar{J}$ [13]. Therefore, some special means have been applied to provide steady-state error-free FFD modeling with reasonably low $\bar{J}$.
2.2. Normalized finite fractional difference $[13,18]$.

Definition 2. Let the fractional difference (FD) be defined as in Eqs. (1) to (3) and the finite fractional difference (FFD) be defined as in Definition 1. Then the normalized finite fractional difference (NFFD) is defined as

$$
\Delta_{N}^{\alpha} x(t, J)=x(t)+\frac{1}{N} \sum_{j=1}^{J} P_{j}(\alpha) x(t) q^{-j},
$$

where $N=N(\bar{J})$ is the normalizing factor.

The results below give the main properties of NFFD.

Lemma 1. Let NFFD be defined as in Definition 2. Then steady-state error-free modeling with Eq. (5) can be provided by the selection

$$
N=-\sum_{j=1}^{\bar{J}} P_{j}(\alpha) .
$$

Lemma 2. Let $N=N(\bar{J})$ as in Eq. (6). Then

$$
\lim _{\bar{J} \rightarrow \infty} N(\bar{J})=1 .
$$

The asymptotic properties of the NFFD as $t \rightarrow \infty$ and $\bar{J} \rightarrow \infty$ are summarized in $[13,18]$. Recursive versions of NFFD, also for time-varying order $\alpha=\alpha_{t}$, have been presented in [13]. Powerful modifications to NFFD, called Adaptive NFFD and Perfect NFFD, have also been introduced in [13], with the former one improved in [34].

\section{State space model}

Consider a discrete-time state space LTI system described by the (constant-order) fractional model

$$
\begin{gathered}
\Delta^{\alpha} x(t+1)=A_{f} x(t)+B u(t), \\
y(t)=C x(t)+D u(t),
\end{gathered}
$$

where $x(t) \in \Re^{n}, u(t) \in \Re^{n_{u}}$ and $y(t) \in \Re^{n_{y}}$ are the state, input and output vectors, respectively, $A_{f} \in \Re^{n \times n}$, $B \in \Re^{n \times n_{u}}, C \in \Re^{n_{y} \times n}$ and $D \in \Re^{n_{y} \times n_{u}}$. Without loss of generality we will assume in the sequel that the initial vector $x_{0}$ is zero, especially that we will operate on finite-memory FD approximations that do not trace back to $x_{0}$.

Note that $A_{f}=A-I$, with $A \in \Re^{n \times n}$ representing a discrete-time state space system in a 'regular' form (with $\alpha=1)$ and $I \in \Re^{n \times n}$ is the identity matrix. Accounting that the FD is calculated by formula (1), equation (8) can be presented in the following form [15]

$$
\begin{gathered}
x(t+1)=\left(A_{f}+\alpha I\right) x(t) \\
-\sum_{j=2}^{t+1} P_{j}(\alpha) x(t-j+1)+B u(t) .
\end{gathered}
$$

Using the Definition 1 and Definition 2 we can jointly present FD/FFD/NFFD-based discrete-time state equations as

$$
\begin{gathered}
x(t+1)=\left(A_{f}+\frac{\alpha}{N} I\right) x(t) \\
-\frac{1}{N} \sum_{j=2}^{J} P_{j}(\alpha) x(t-j+1)+B u(t),
\end{gathered}
$$


with $N=N(\bar{J})$ defined as in Definition 2 and $J$ redefined as $J=\min (t+1, \bar{J})$.

Note that Eq. (11) can be considered the most general fractional-difference state equation including the NFFD one, FFD one (for $N=1$ ) and FD one (for $\bar{J} \rightarrow \infty$ implying $N \rightarrow 1)$.

Remark 2. Possible accounting for the sampling period $T$ (when transferring from a continuous-time derivative to the discrete-time difference) results in the substitutions $A_{f} \rightarrow$ $A_{f} T^{\alpha}$ and $B \rightarrow B T^{\alpha}$ in Eqs. (10) and (11) [15].

\subsection{Steady-state accuracy [13]}

Theorem 1. Let the steady-state output error for the FFD/NFFD-based state-space model with respect to the FD one be defined as

$$
\epsilon_{s s}=\lim _{t \rightarrow \infty}\left\{\epsilon_{y}(t)=y_{\mathrm{NFD}}(t)-y_{\mathrm{FD}}(t)\right\},
$$

where $y_{\mathrm{NFD}}(t)$ and $y_{\mathrm{FD}}(t)$ are the outputs of the FFD/NFFDbased and FD-based state-space models, respectively. Additionally, let $A_{f}$ be nonsingular. Then

$$
\epsilon_{s s}=C\left(F-A_{f}\right)^{-1} F A_{f}^{-1} B u_{s s},
$$

where $u_{s s}$ is the steady state input and

$$
F=\left[1+\frac{1}{N} \sum_{j=1}^{\bar{J}} P_{j}(\alpha)\right] I
$$

with $N=N(\bar{J})$ as in Lemma 1 for the NFFD-based system and $N=1$ for the FFD-based one.

It is clear now that steady-state error-free output modeling can be obtained for the NFFD-based system only. In fact, the matrix $F$ is zero in that case.

\section{Stability}

4.1. NFFD-based system. Our preliminary NFFD stability result is now recalled as

Theorem 2. [13] The NFFD-based discrete-time state equation (11), with $\alpha \in(0,2)$ and $N$ selected as in Lemma 1 , is asymptotically stable if

$$
\|\underline{A}\|<\phi(\alpha)
$$

where $\|$.$\| is a norm of a matrix, \underline{A}=A_{f}+\frac{\alpha}{N} I, \phi(\alpha)=\frac{\alpha}{N}$ for $\alpha \in(0,1)$ and $\phi(\alpha)=2-\frac{\alpha}{N}$ for $\alpha \in(1,2)$.

Interestingly, the stability condition (15) for the NFFDbased state space system with the normalizing factor selected according to Lemma 1 includes both that for the FD-based one (compare Monje et al., 2010), in which case $\bar{J} \rightarrow \infty$ implying $N \rightarrow 1$, and that for the FFD-based one $(N=1)$ as well as that for the "regular" difference ( $\alpha=1$ and $N=1$ ).

Remark 3. For the FFD-based system, the result of Theorem 2 is similar to those of [10] and [15]. More general but computationally involving stability results for the FFD-based system have been given in Refs. [26,27].
In the sequel, all the illustrating examples will be processed in the Matlab environment.

Example 1. Consider the NFFD-based discrete-time state space system with

$$
A_{f}=\left[\begin{array}{ll}
-0.10224 & 0.31921 \\
-0.24145 & 0.31286
\end{array}\right]
$$

The actual stability area and that obtained from Theorem 2 are presented in Fig. 1 (note: The actual stability area is determined from heuristic BIBO stability experiments and verified by the analytical result of the forthcoming Theorem 3 ).

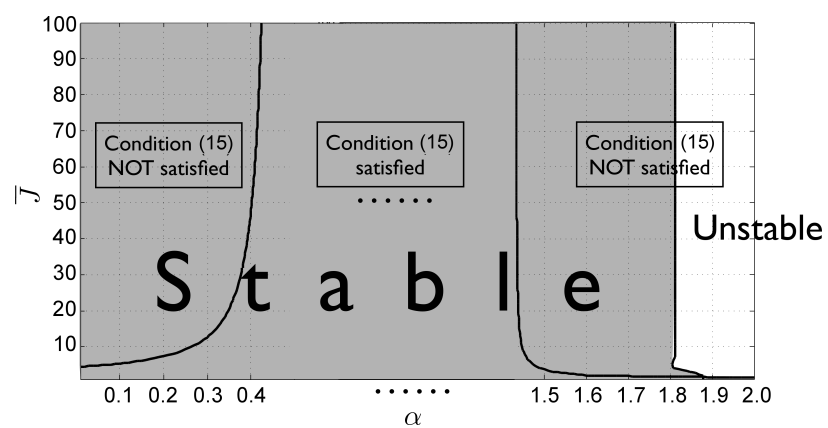

Fig. 1. The actual stability area vs. that of Theorem 2

It can be seen that the conservative condition (15) covers only some $50 \%$ of the actual stability area and it strongly depends on $\alpha$. Moreover, for specific values of $\alpha$ (and $\bar{J}$ ) the actual NFFD/FFD system can be stable whereas the condition (15) is never satisfied.

The above Example shows that it is of vital interest to seek for necessary and sufficient conditions of the asymptotic stability. Here is the first, new, original result in the field.

Theorem 3. The NFFD-based discrete-time state equation (11) with $\alpha \in(0,2)$ is asymptotically stable if and only if all the roots of the characteristic equation

$$
\operatorname{det}\left(I z^{\bar{J}}-\underline{A} z^{\bar{J}-1}+\sum_{j=2}^{\bar{J}} \beta_{j} z^{\bar{J}-j}\right)=0
$$

where $z \in \mathcal{C}, \underline{A}=A_{f}+\frac{\alpha}{N} I$ and $\beta_{j}=\frac{1}{N} P_{j}(\alpha) I, j=2, \ldots \bar{J}$, are strictly inside the unit circle.

Proof. See Appendix A.

Remark 4. The result for an FFD-based state space equation is immediately obtained by setting $N=1$.

Remark 5. Note that a rather complicated proof of this new fundamental stability result (tracing back to the original $\widetilde{A}$ formulation) can be easily verified. In fact, the $z$-domain solution to the state equation (11), under the condition $x_{0}=0$, can be obtained as

$$
X(z)=\left(z I-\underline{A}+\frac{1}{N} \sum_{j=2}^{\bar{J}} P_{j}(\alpha) z^{-j+1}\right)^{-1} B U(z)
$$

which leads immediately to the characteristic equation (16). 
Note that the above Remark 5 can stand in fact as proof of Theorem 3, so that proof of Appendix A might be considered unnecessary. However, we strongly emphasize that an original idea of relying the stability proof on the matrix $\widetilde{A}[14,15]$ is worth of further dissemination. The idea is effectively used in the stability analysis of a combined, FFD/Laguerre-based system [35] employing the new FD modeling concept intimated in Ref. [36].

4.2. FD-based system. Related to Theorem 3, a new result for an FD-based state-space system can be obtained as

Theorem 4. The FD-based discrete-time state equation (10) with $\alpha \in(0,2)$ is asymptotically stable if and only if all the roots of the characteristic equation

$$
\operatorname{det}\left[z\left(1-z^{-1}\right)^{\alpha} I-A_{f}\right]=0
$$

are strictly inside the unit circle.

Proof. See Appendix B.

Remark 6. It is interesting to note that for FD-based state space equation (11), with $x_{0}=0$ and $\bar{J} \rightarrow \infty$ implying $N \rightarrow 1$, we have

$$
X(z)=\left[z\left(1-z^{-1}\right)^{\alpha} I-A_{f}\right]^{-1} B u(z) .
$$

Example 2. Consider the NFFD/FFD/FD-based discrete-time state space system with

$$
A_{f}=\left[\begin{array}{cc}
0.6 & -1.45 \\
1 & -1
\end{array}\right] \text {. }
$$

The stability areas obtained from Theorems 3 and 4 are presented in Fig. 2.

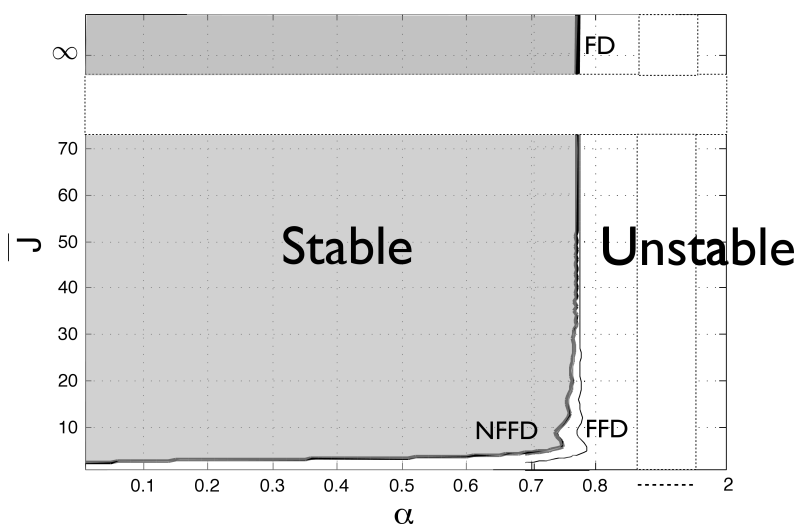

Fig. 2. Stability areas obtained from Theorems 3 and 4

It is interesting to note from Fig. 2 that the NFFDbased system is unstable for very low $\bar{J}$. For higher $\bar{J}$, the NFFD/FFD-based system is unstable for $\alpha$ exceeding some critical value. For $\bar{J} \rightarrow \infty$, the stability condition for the FD-based system is $\alpha<0.7749$. It is interesting to note that this condition cannot be "seen" by Theorem 2 under $\bar{J} \rightarrow \infty$ and $N=1$. In fact, the condition (15) is never satisfied for the specific system of Example 2.
4.3. Coordinate transformations. It is interesting now to pursue a stability area for FD-based systems in a transformed complex domain with respect to eigenvalues of the matrix $A_{f}$ (or $A$ ). We can provide yet another, new result in this respect. Note that the characteristic Eq. (18) can be rewritten as $\operatorname{det}\left(w I-A_{f}\right)=0$, where

$$
w=z\left(1-z^{-1}\right)^{\alpha},
$$

is the (nonlinear) coordinate transformation, mapping the unit circle into a $w$-domain stability contour for FD (Fig. 3). Alternatively, referring to the integer-order state matrix $A=$ $A_{f}+I$, whose well-known stability condition can be a reference, we can introduce another coordinate transformation

$$
v=z\left(1-z^{-1}\right)^{\alpha}+1=f_{\alpha}(z)
$$

which, for a specific $\alpha$, maps the unit circle into a $v$-domain stability contour for FD (Fig. 3).

Remark 7. Note that, unsurprisingly, the $v$-domain contour reduces to the $z$-domain one for $\alpha=1$.

Since it is impossible to analytically determine an inverse $z=f_{\alpha}^{-1}(v)$, we find the $v$-domain stability contour for FD in a numerical way.

To this end, recall the $z$-domain stability contour, which is the unit circle, for which the modulus $|z|=1$, that is $z=e^{i \varphi}$, where $\varphi=\arg (z)$. Thus,

$$
v=e^{i \varphi}\left(1-e^{-i \varphi}\right)^{\alpha}+1
$$

or

$$
w=e^{i \varphi}\left(1-e^{-i \varphi}\right)^{\alpha}
$$

which can be plotted for a specific $\alpha$ within the range $0 \leq$ $\varphi \leq 2 \pi$. The obtained $v$-domain ( $w$-domain) vs. $z$-domain stability contours for $\alpha=0.6$ are shown in Fig. 3 .

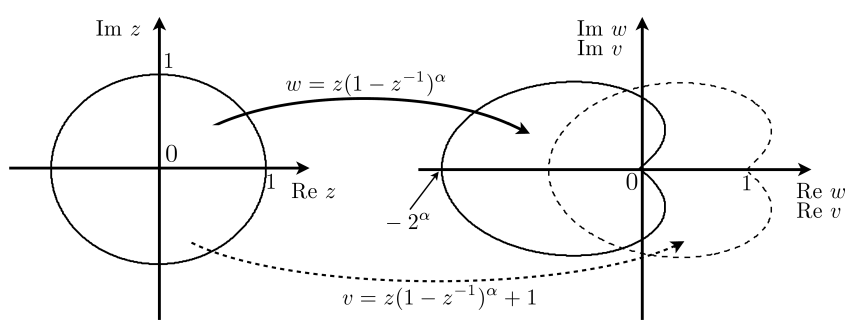

Fig. 3. Illustration of coordinate transformations (20) and (21) for $\alpha=0.6$

It is interesting that the specific value $\left(-2^{\alpha}\right)$ for $\operatorname{Im} w=0$ (or $1-2^{\alpha}$ for $\operatorname{Im} v=0$ ) can be obtained analytically. We will show that, for $\alpha \in(0,1)$, the interior of the unit circle is mapped by the transformations (20) or (21) into the interior of the FD stability contour, but this does not necessarily hold true for $\alpha \in(1,2)$.

It is also of interest to seek for a 3D illustration of the $w$ domain stability contours, with $\alpha$ parametrized (i.e. $\alpha=$ par). The result for selected values of $\alpha$ is presented in Fig. 4. 


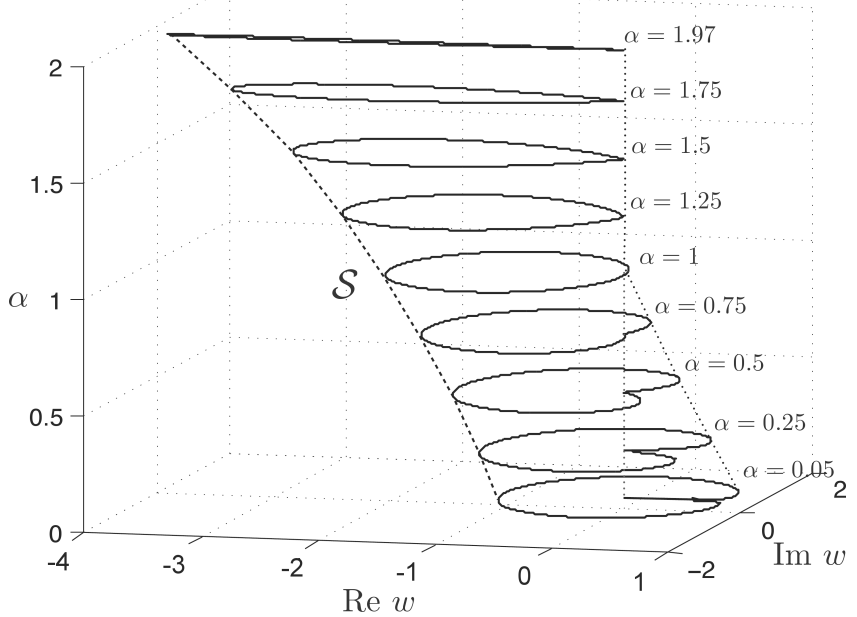

Fig. 4. Plot of (the sections of) the FD stability solid for the specific values of $\alpha$

The obtained 3D geometric shape constitutes the general stability boundary for the FD-based state equation. We shortly call the 3D geometric shape $\mathcal{S}$ as "the FD stability solid". We are now in a position to formulate yet another new stability result.

Theorem 5. Consider the FD-stability solid related with the coordinate transformation (21), with $\alpha=$ par. The FD-based discrete-time state Eq. (10) with $\alpha \in(0,2)$ is asymptotically stable if and only if all the roots of the characteristic equation

$$
\operatorname{det}[v I-A]=0
$$

that is all eigenvalues of $A$, are strictly inside the FD stability solid.

Proof. See Appendix C.

Since $\operatorname{det}(v I-A)=\operatorname{det}\left(w I-A_{f}\right)$, Theorem 5 can be easily reformulated for the mapping (20) to obtain.

Theorem 6. Consider the FD stability solid $\mathcal{S}$ related with the coordinate transformation (20), with $\alpha=$ par. The FD-based discrete-time state equation (10) with $\alpha \in(0,2)$ is asymptotically stable if and only if all the roots of the characteristic equation

$$
\operatorname{det}\left[w I-A_{f}\right]=0
$$

that is all eigenvalues of $A_{f}$, are strictly inside the FD stability solid.

The boundary surface related with the FD stability solid can be calculated once with some resolution step for $\alpha$ and kept stored in a data base for future use, or, more practically, the boundary contour can be immediately calculated for a specific $\alpha$ according to equation (22) or (23).

Remark 8. We continue with the mapping (20) as it is shown that, rather surprisingly, the presence of the unity in the transformation (21) precludes some simple analytical manipulations in the complex $v$-domain.

Remark 9. The stability domain related with the mapping (20) has also been discussed in Ref. [8] for $\alpha=1 / d, d=1,2, \ldots$.
Example 3. Consider the FD-based discrete-time state equation with

$$
A_{f}=\left[\begin{array}{cc}
0.2 & -0.5121 \\
1 & -1
\end{array}\right]
$$

whose eigenvalues are $z_{1}=-0.4-0.39 i, z_{2}=-0.4+0.39 i$ as depicted with the asterisks in Fig. 5, which is a horizontal cross-section of the stability solid $\mathcal{S}$ of Fig. 4 for specific $\alpha$ s.

Testing for the asymptotic stability according to Theorem 4 or 6 reveals that the system is stable e.g. for $\alpha=0.7$ and $\alpha=1.2$, but unstable for $\alpha=1.5$ (Fig. 5).

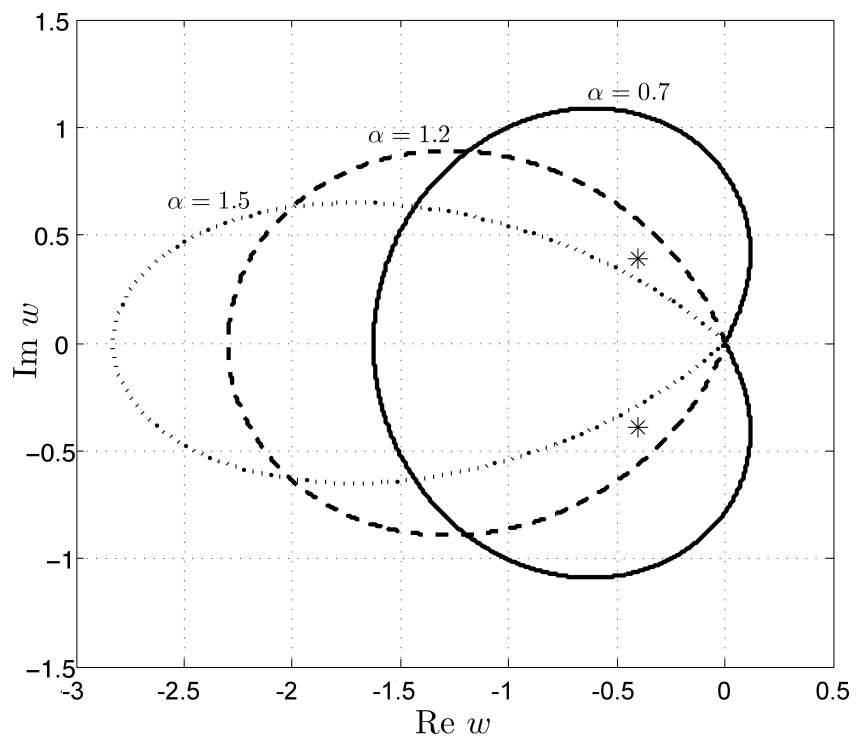

Fig. 5. Plots of FD stability contours for the specific values of $\alpha$

4.4. NFFD/FFD/FD-based systems revisited. We are in a position now to present a general stability result valid for NFFD, FFD and FD-based systems.

Theorem 7. The NFFD/FFD/FD-based discrete-time state equation (11) with $\alpha \in(0,2)$ is asymptotically stable if and only if all the roots of the characteristic equation

$$
\operatorname{det}\left[w I-A_{f}\right]=0
$$

that is all eigenvalues of $A_{f}$, are strictly inside the stability solid

$$
\mathcal{S}=\left\{e^{i \varphi} \Psi(\varphi) ; 0<\alpha<2 ; 0 \leq \varphi \leq 2 \pi\right\}
$$

and

i) $\Psi(\varphi)=1+\frac{1}{N} \sum_{j=1}^{\bar{J}} P_{j}(\alpha) e^{-i j \varphi}$ for NFFD,

ii) $\Psi(\varphi)$ as above with $N=1$ for FFD,

iii) $\Psi(\varphi)=\left(1-e^{-i \varphi}\right)^{\alpha}$ for FD.

Proof. The result follows immediately from Theorems 3, 4 and 6.

Remark 10. Again, for specific $\alpha$ we can operate on a specific stability contour, that is a specific section of the stability solid for $\alpha=$ par. And again, Theorem 7 can be easily reformulated to include $v$ and $A$. 
Remark 11. The considered NFFD/FFD/FD stability contours are quite regular in shape so that a selection of increments for $\varphi$, with $0 \leq \varphi \leq 2 \pi$, need not be "dense".

Note that our result for FFD is essentially simpler from the computational viewpoint than the necessary and sufficient conditions of $[10,15]$, where expanding the size of the matrix $\widetilde{A}$ (see proof of Theorem 3 ) for higher $\bar{J}$ might lead to prohibitive numerical problems e.g. in the Matlab environment. Also, execution times for even admissible values of $\bar{J}$ were dramatically high in $[10,15]$ as compared with our result which operates on the simple calculation of the contour (27) (in addition to eigenvalues of the matrix $A_{f}$ ). We have not experienced any problems under Matlab, even for $\bar{J}=100,000$ which is in fact unreasonably and unnecessarily high.

Example 4. Consider the NFFD/FFD/FD-based discrete-time state space system with $\alpha=0.5$ and

$$
A_{f}=\left[\begin{array}{cc}
0.58 & -0.54 \\
1 & -1
\end{array}\right]
$$

whose eigenvalues are $z_{1}=0.08, z_{2}=-0.5$.

The stability areas obtained from Theorem 7 under the specifications i), ii) and iii) are presented in Fig. 6. Theorem 7 reveals that FD and NFFD-based systems $(\bar{J}=30)$ are unstable but the FFD-based system is stable. The example selection for $A_{f}$ serves illustrating a rather typical case where inferring on the asymptotic stability of FFD-based systems may sometimes be less reliable than for the NFFD-based ones.

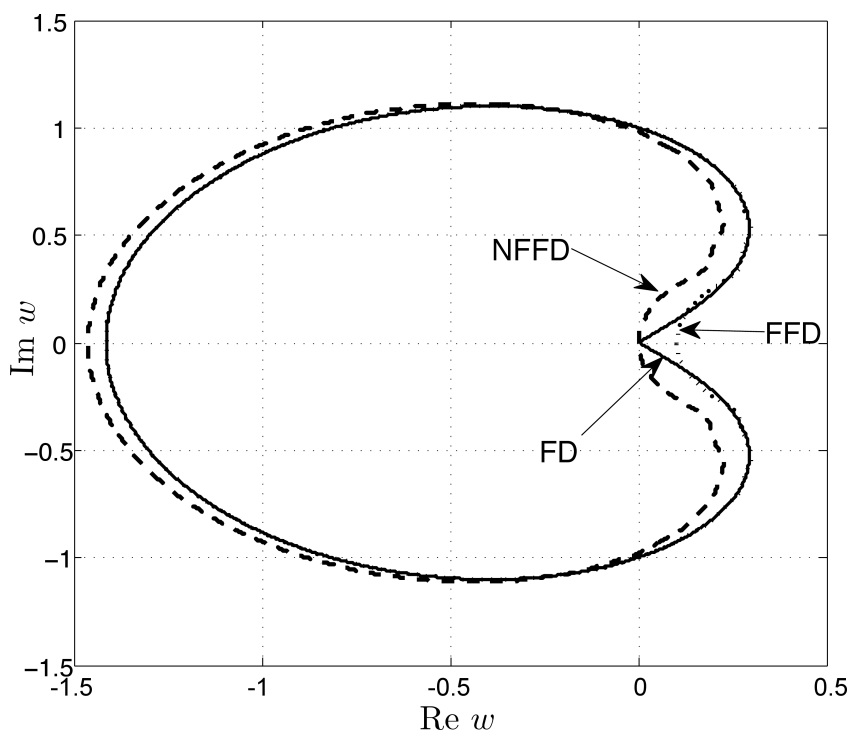

Fig. 6. Plots of NFFD/FFD/FD stability contours for $\alpha=0.5$

\section{Conclusions}

Previously formulated necessary and sufficient stability conditions for FD-based state space LTI systems have suffered from computational ineffectiveness. In Part I of the paper, new compact results have been formulated, referring the stability of FD/FFD/NFFD-based state space systems to the roots of certain characteristic equations, in particular eigenvalues of some low-dimensional matrix. This has led to essential computational savings as compared to previous stability results.

Our final contribution in Part I has been the introduction of a unified framework to analyze FD/FFD/NFFD-based LTI state space systems in the complex domain. The framework directly leads in Part II to the presentation of an original stability criterion for the FD-based systems as well as new, simple numerical procedures for an approximate testing the stability of FFD/NFFD-based systems.

\section{Appendix A. Proof of Theorem 3}

Equation (11) can be presented by the extended state equation $[14,15]$, with

$$
\widetilde{A}=\left[\begin{array}{ccccc}
\underline{A} & -\beta_{2} & \ldots & -\beta_{\bar{J}-1} & -\beta_{\bar{J}} \\
I & 0 & \ldots & 0 & 0 \\
0 & I & \ldots & 0 & 0 \\
\vdots & \vdots & \ddots & \vdots & \vdots \\
0 & 0 & \ldots & I & 0
\end{array}\right],
$$

where $\underline{A}=A_{f}+\frac{\alpha}{N} I$ and $\beta_{j}=\frac{1}{N} P_{j}(\alpha) I, j=2, \ldots \bar{J}$. The matrix $z I-\widetilde{A}$ is now

$$
=\left[\begin{array}{cccccc}
z I-\underline{A} & \beta_{2} & \beta_{3} & \ldots & \beta_{\bar{J}-1} & \beta_{\bar{J}} \\
-I & z I & 0 & \ldots & 0 & 0 \\
0 & -I & z I & \ldots & 0 & 0 \\
\vdots & \vdots & \vdots & & \vdots & \vdots \\
0 & 0 & 0 & \ldots & -I & z I
\end{array}\right] .
$$

We consider the consecutive columns of the matrix $H_{1}$ in the reverse order, that is starting from the last column. Denote the reverse-order columns of that matrix as $C_{j}^{1}, j=\bar{J}, \ldots, 1$. Using the elementary column operation $C_{\bar{J}_{-1}}^{2} \leftarrow C_{\frac{1}{J}-1}^{1}+z^{-1} C_{\bar{J}}^{1}$ on $H_{1}$ we obtain the matrix

$$
\left[\begin{array}{cccccc}
z I-\underline{A} & \beta_{2} & \beta_{3} & \ldots & \beta_{\bar{J}-1}-z^{-1} \beta_{\bar{J}} & \beta_{\bar{J}} \\
-I & z I & 0 & \ldots & 0 & 0 \\
0 & -I & z I & \ldots & 0 & 0 \\
\vdots & \vdots & \vdots & & \vdots & \vdots \\
0 & 0 & 0 & \ldots & 0 & z I
\end{array}\right]
$$

thus getting rid of the submatrix $(-I)$ from the pre-last column of $H_{1}$.

Using consecutively the elementary column operations $C_{j}^{i+1} \leftarrow C_{j}^{i}+z^{-1} C_{j+1}^{i}, i=2, \ldots, \bar{J}-1, j=\bar{J}-2, \ldots, 1$ 
Stability analysis for discrete-time fractional-order LTI state-space systems. Part I.

on the consecutive matrices $H_{i}, i=2, \ldots, \bar{J}$, we arrive at the triangular matrix

$$
H_{\bar{J}}=\left[\begin{array}{ccccc}
z I-\underline{A}+S_{1} & S_{2} & S_{3} & \ldots & S_{\bar{J}}=\beta_{\bar{J}} \\
0 & z I & 0 & \ldots & 0 \\
0 & 0 & z I & \ldots & 0 \\
\vdots & \vdots & \vdots & \ddots & \vdots \\
0 & 0 & 0 & \ldots & z I
\end{array}\right]
$$

where

$$
S_{1}=\sum_{j=2}^{\bar{J}} \beta_{j} z^{-j+1} \quad \text { and } \quad S_{i}=\sum_{j=i}^{\bar{J}} \beta_{j} z^{-j+i}, \quad i=2, \ldots, \bar{J} .
$$

The essence of the above matrix transformations is that the elementary column operations

$$
\begin{gathered}
C_{j}^{i+1} \leftarrow C_{j}^{i}+z^{-1} C_{j+1}^{i}, \\
i=1, \ldots, \bar{J}-1, \quad j=\bar{J}-1, \ldots, 1,
\end{gathered}
$$

do not change the determinants of the matrices $H_{1}$ to $H_{\bar{J}}$ $[37,38]$, so that, finally, the determinant of the triangular matrix $H_{\bar{J}}$ is of form

$$
\begin{gathered}
\operatorname{det}(z I-\widetilde{A})=\operatorname{det}\left[I z^{\bar{J}-1}\left(z I-\underline{A}+S_{1}\right)\right] \\
=\operatorname{det}\left[I z^{\bar{J}}-\underline{A} z^{\bar{J}-1}+\sum_{j=2}^{\bar{J}} \beta_{j} z^{\bar{J}-j}\right]
\end{gathered}
$$

and the result follows.

\section{Appendix B. Proof of Theorem 4}

Rewrite the matrix in equation (16) for $N=1$ and obtain

$$
\begin{gathered}
z^{\bar{J}-1}\left[\left(I z \left(1+P_{1}(\alpha) z^{-1}+P_{2}(\alpha) z^{-2} \ldots\right.\right.\right. \\
\left.\left.+P_{\bar{J}}(\alpha) z^{-\bar{J}}\right)-A_{f}\right]
\end{gathered}
$$

with $P_{1}(\alpha)=-\alpha$. Now, for $\bar{J} \rightarrow \infty$, using the extended Newton binomial

$$
(a+b)^{\alpha}=\sum_{j=0}^{\infty}\left(\begin{array}{l}
\alpha \\
j
\end{array}\right) a^{\alpha-j} b^{j}
$$

and accounting for the fact that $\sum_{j=0}^{\infty} P_{j}(\alpha) z^{-j}$ is the binomial expansion for $a=1$ and $b=-z^{-1}$ we can write that

$$
\begin{gathered}
\lim _{\bar{J} \rightarrow \infty}\left(1+P_{1}(\alpha) z^{-1}+P_{2}(\alpha) z^{-2} \ldots+P_{\bar{J}}(\alpha) z^{-\bar{J}}\right) \\
=\left(1-z^{-1}\right)^{\alpha}
\end{gathered}
$$

leading, together with (33), to the characteristic equation (18).

\section{Appendix C. Proof of Theorem 5}

Proof of Theorem 5 parallels that for the celebrated Cauchy's Argument Principle-based Nyquist Stability Criterion [39].
The characteristic equation (24) can be written as

$$
\operatorname{det}(v I-A)=\left(v-\lambda_{1}\right)\left(v-\lambda_{2}\right) \ldots\left(v-\lambda_{n}\right)=0,
$$

where $\lambda_{i}, i=1, \ldots, n$, are the eigenvalues of $\mathrm{A}$. The system is asymptotically stable iff all the elements $v-\lambda_{i}, i=1, \ldots, n$, do not generate unstable poles in the $z$-domain. Assuming that $\bar{J} \rightarrow \infty$ we obtain the meromorphic function $v(z)$ (compare Proof of Theorem 4)

$$
\begin{gathered}
v(z)=z\left(1-z^{-1}\right)^{\alpha}+1 \\
=\lim _{\bar{J} \rightarrow \infty} \frac{z^{\bar{J}}+\sum_{j=1}^{\bar{J}} P_{j}(\alpha) z^{\bar{J}-j}+z^{\bar{J}-1}}{z^{\bar{J}-1}} .
\end{gathered}
$$

Now, consider a simple closed positively oriented contour $D$ (Fig. 7) whose interior domain is $\underline{C}=\mathcal{C} \backslash\left\{r e^{i \varphi}, 0 \leq\right.$ $r<1,0 \leq \varphi \leq 2 \pi\}$ such that for $z \in C, v(D) \neq 0$ and $v(D) \neq \infty$. Then by virtue of the Argument Principle and Rouche's Theorem [39]

$$
W\left(v(D), \lambda_{i}\right)=\frac{1}{2 \pi i} \oint_{D} \frac{v^{\prime}(z)}{v(z)-\lambda_{i}} d z=n_{z}-n_{p}
$$

where $W\left(v(D), \lambda_{i}\right)$ is the winding number of $v(D)$ about $\lambda_{i}$, that counts the number of times the curve $v(D)$ winds around the point $\lambda_{i}$, and $n_{z}$ and $n_{p}$ denote the number of zeros and poles of $v(z)-\lambda_{i}$ inside the contour $D$, respectively. Immediately from Eq. (37) we have $n_{p}=0$. The contour $v(D)$ is presented in Fig. 8 and we can observe that when $\lambda_{i}$ is inside the area bounded by $e^{i \varphi}\left(1-e^{-i \varphi}\right)^{\alpha}+1$, then $W\left(v(D), \lambda_{i}\right)=0$ so the zeros of Eq. (37) are not enclosed by the contour $D$ and they are inside the area bounded by $e^{i \varphi}$.

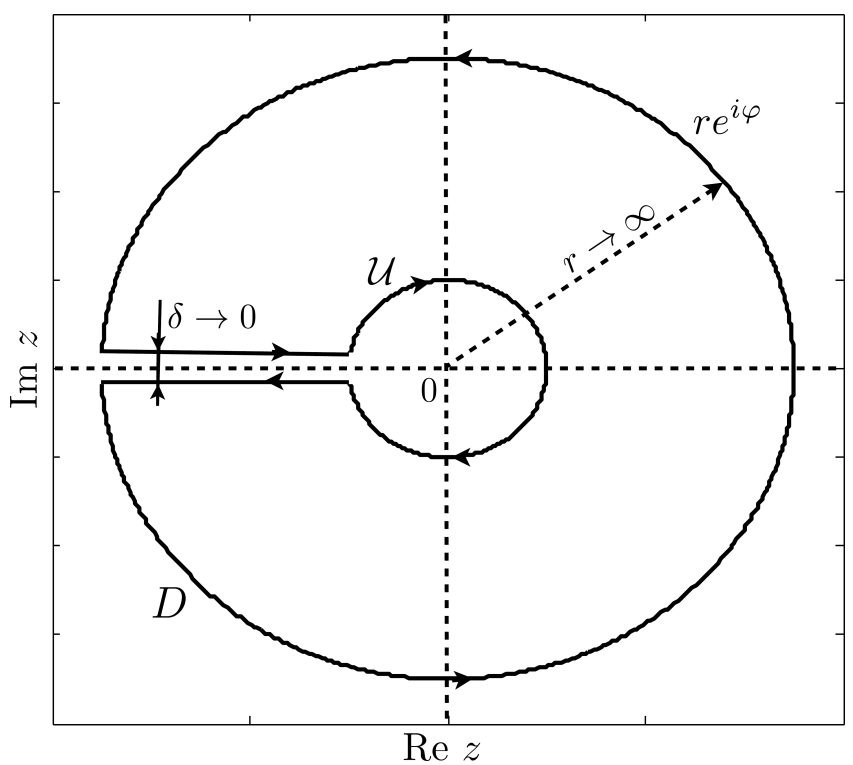

Fig. 7. Simple closed positively oriented contour $D$ (including $\mathcal{U}$ ) 


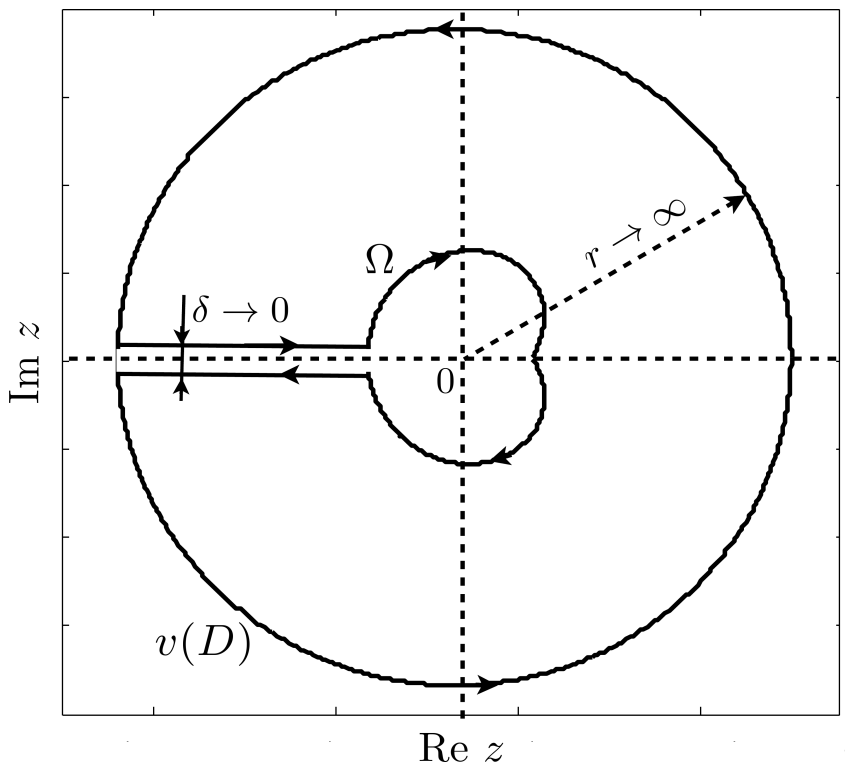

Fig. 8. The contour of $v(D)$ (including $\Omega$ )

It is essential that the inference of $\lambda_{i}$ is directed from the interior of $\Omega=\left\{e^{i \varphi}\left(1-e^{i \varphi}\right)^{\alpha}+1,0 \leq \varphi \leq 2 \pi\right\}$, with $\Omega \subset v(D)$, to the interior of $\mathcal{U}=\left\{e^{i \varphi}, 0 \leq \varphi \leq 2 \pi\right\}$, with $\mathcal{U} \subset D$, and not vice versa. This means that the stability area involves the whole interior of $\Omega$, but this must not necessarily be mapped to by the whole interior of $\mathcal{U}$, the case being characteristic for $\alpha \in(1,2)$. Well, for $\alpha \in(0,1)$ the inference operates on the whole interiors of both $\Omega$ and $\mathcal{U}$. Anyway, the proof is valid for $\alpha \in(0,2)$.

\section{REFERENCES}

[1] H. Delavari, A. Ranjbar, R. Ghaderi, and S. Momani, "Fractional order control of a coupled tank", Nonlinear Dynamics 61 (3), 383-397 (2010).

[2] I. Petráš and B. Vinagre, "Practical application of digital fractional-order controller to temperature control", Acta Montanistica Slovaca 7 (2), 131-137 (2002).

[3] D. Riu, N. Retiére, and M. Ivanes, "Turbine generator modeling by non-integer order systems", IEEE Int. Conf. on Electric Machines and Drives, Cambridge 1, 185-187 (2001).

[4] V. Zaborowsky and R. Meylaov, "Informational network traffic model based on fractional calculus", Proc. Int. Conf. Info-tech and Info-net (ICII'2001) 1, 58-63 (2001).

[5] C.H. Lubich, "Discretized fractional calculus", SIAM J. on Mathematical Analysis 17 (3), 704-719 (1986).

[6] M.D. Ortigueira, "Introduction to fractional linear systems ii: Discrete-time case", IEE Proc. on Vision, Image and Signal Processing 147 (1), 71-78 (2000).

[7] P. Ostalczyk, "The non-integer difference of the discrete-time function and its application to the control system synthesis", Int. J. Systems Science 31 (12), 1551-1561 (2000).

[8] P. Ostalczyk, "Equivalent descriptions of a discrete-time fractional-order linear system and its stability domains", Int. J. Applied Mathematics and Computer Science 22 (3), 533-538 (2012).

[9] I. Petráš, L. Dorčák, and I. Koštial, "The modelling and analysis of fractional-order control systems in discrete domain", Proc. Int. Carpatian Control Conf. 1, 257-260 (2000).
[10] A. Dzieliński and D. Sierociuk, "Stability of discrete fractional order state-space systems", J. Vibration and Control 14 (9-10), 1543-1556 (2008).

[11] T. Kaczorek, "Practical stability of positive fractional discretetime linear systems", Bull. Pol. Ac.: Tech. 56 (4), 313-317 (2008).

[12] D. Sierociuk and A. Dzieliński, "Fractional Kalman filter algorithm for states, parameters and order of fractional system estimation", Int. J. Applied Mathematics and Computer Science 16 (1), 101-112 (2006).

[13] R. Stanisławski and K.J. Latawiec, "Normalized finite fractional differences: the computational and accuracy breakthroughs", Int. J. Applied Mathematics and Computer Science 22 (4), 907-919 (2012).

[14] M. Busłowicz and T. Kaczorek, "Simple conditions for practical stability of positive fractional discrete-time linear systems", Int. J. Applied Mathematics and Computer Science 19 (2), 263-269 (2009).

[15] C. Monje, Y. Chen, B. Vinagre, D. Xue, and V. Feliu, Fractional-order Systems and Controls, Springer-Verlag, London, 2010.

[16] I. Podlubny, Fractional Differential Equations, Academic Press, Orlando, 1999.

[17] R. Stanisławski, "Identification of open-loop stable linear systems using fractional orthonormal basis functions", Proc. 14th Int. Conf. on Methods and Models in Automation and Robotics 1, 935-985 (2009).

[18] R. Stanisławski, W. Hunek, and K.J. Latawiec, "Finite approximations of a discrete-time fractional derivative", 16th Int. Conf. on Methods and Models in Automation and Robotics 1, 142145 (2011).

[19] R. Stanisławski and K.J. Latawiec, "Modeling of open-loop stable linear systems using a combination of a finite fractional derivative and orthonormal basis functions", Proc. 15th Int. Conf. on Methods and Models in Automation and Robotics 1, 411-414 (2010).

[20] I. Kheirzad, M.S. Tavazoei, and A.A. Jalali, "Stability criteria for a class of fractional order systems", Nonlinear Dynamics 61 (1-2), 153-161 (2010).

[21] C.-p. Li and Z.-g. Zhao, "Asymptotical stability analysis of linear fractional differential systems", J. Shanghai University (English Edition) 13 (3), 197-206 (2009).

[22] D. Matignon, "Stability results for fractional differential equations with applications to control processing", Computational Engineering in Systems Applications 2, 963-968 (1996).

[23] D. Matignon, "Stability properties for generalized fractional differential systems", ESAIM: Proc. 5, 145-158 (1998).

[24] I. Petrás, "Stability of fractional-order systems with rational orders: A survey", Fractional Calculus and Applied Analysis 12 (3), 269-298 (2009).

[25] J. Sabatier, M. Moze, and C. Farges, "On stability of fractional order systems", Third IFAC Workshop on Fractional Differentiation and Its Applications FDA'08 1, CD-ROM (2008).

[26] S. Guermah, S. Djennoune, and M. Bettayeb, "A new approach for stability analysis of linear discrete-time fractional-order systems", New Trends in Nanotechnology and Fractional Calculus Applications 1, 151-162 (2010).

[27] S.B. Stojanovic and D.L. Debeljkovic, "Simple stability conditions of linear discrete time systems with multiple delay", Serbian J. Electrical Engineering 7 (1), 69-79 (2010).

[28] M. Busłowicz, "Robust stability of positive discrete-time linear systems of fractional order", Bull. Pol. Ac.: Tech. 58 (4), 
Stability analysis for discrete-time fractional-order LTI state-space systems. Part I.

$567-572(2010)$

[29] T. Kaczorek, "Practical stability and asymptotic stability of positive fractional 2d linear systems", Asian J. Control 12 (2), 200-207 (2010).

[30] T. Kaczorek, "New stability tests of positive standard and fractional linear systems", Circuits and Systems 2 (4), 261-268 (2011).

[31] T. Kaczorek, Selected Problems of Fractional Systems Theory, Springer-Verlag, Berlin, 2011.

[32] K. Miller and B. Ross, An Introduction to the Fractional Calculus and Fractional Differential Equations, Willey, New York, 1993.

[33] K. Oldham and J. Spanier, The Fractional Calculus, Academic Press, Orlando, 1974.

[34] K.J. Latawiec, R. Stanisławski, W.P. Hunek, and M. Lukaniszyn, "Adaptive finite fractional difference with a timevarying forgetting factor", Proc. 17th Int. Conf. on Meth- ods and Models in Automation and Robotics 1, 64-69 (2012).

[35] R. Stanisławski, Advances in Modeling of Fractional Difference Systems - New Accuracy, Stability and Computational Results, Opole University of Technology Press, Opole, 2013, (to be published).

[36] R. Stanisławski, "New Laguerre filter approximators to the Grünwald-Letnikov fractional difference", Mathematical Problems in Engineering 2012, 732917 (2012).

[37] G. Shilov, Linear Algebra, ser. Dover Books on Advanced Mathematics, Dover Publications, Mineola, 1977.

[38] B.L. Willis, "Eigenvalues by row operations", University of Nebraska at Kearney, Karney, [Online]. Available: http://www.unk.edu/uploadedFiles/facstaff/profiles/willisb/eigens-by-row(1).pdf (2005).

[39] R. Lopez, Advanced Engineering Mathematics, Addison Wesley Publishing Company, Boston, 2001. 